

\title{
Pain assessment in older adults with dementia: An exploratory pilot study of paramedic students' perceptions of the utility of two validated assessment tools
}

Peter V Lucas BA, MSocSc, PhD, is a Research Fellow'; Ron Mason MA, is a Junior Research Fellow²; Michael Annear MAppSc, PhD, is a Research Fellow²; Wayne Harris BHSc(Paramedic), GradCertEd, is a Lecturer ${ }^{1}$; Michael $\mathrm{J}$ McCall MMedSc, is Principle Educator; ; Andrew Robinson RN, DipAppSc(Nurs), BAppScNurs (Ed), MNS, PhD, is Professor of Aged Care Nursing, Co-Director²; Fran Mclnerney RN, BAppSci, MA, PhD, is Professor of Dementia Studies and Education ${ }^{2}$

\author{
Affiliations: \\ ${ }^{1}$ Division of Paramedicine, University of Tasmania \\ ${ }^{2}$ Wicking Dementia Research and Education Centre, University of Tasmania \\ ${ }^{3}$ Simulated Learning Environments, Department of Health and Human Services
}

\section{Abstract}

\section{Introduction}

This paper presents findings from an exploratory pilot study in which undergraduate paramedic students trialled the use of two pain assessment tools as part of an interprofessional learning activity in residential aged care facilities (RACFs). The research sought to gain students' perceptions of the potential utility of the Abbey Pain Scale and PAINAD tools for use by paramedics with people with advanced dementia who have limited ability to communicate.

\section{Methods \\ Thirty-one final year undergraduate paramedic students completed a 5-day clinical placement in four RACFs in Tasmania, Australia. While on placement, students used the two clinically validated pain assessment tools to assess pain in residents with known pain issues, under the supervision of nursing staff and paramedic tutors. A mixed methods approach, utilising a quantitative survey and a qualitative open-ended questionnaire, was adopted to ascertain students' perceptions about the potential for the tools to be used in paramedic practice.}

\section{Results}

The research found that students considered both tools had potential for use in paramedic practice. Feedback from this cohort of students indicated they considered the Abbey Pain Scale was more useful for assessing pain in cognitively impaired older people, although students reported they considered the PAINAD Scale to be more relevant to their future role as paramedics. Recommendations were made by the students for how each of the tools might be adapted to make them more suitable for use by paramedics.

\section{Conclusion}

Forecast increases in the number of people living with dementia, and the changing nature of paramedic practice in the 21st century, means that paramedics are more likely to be called on to assess pain in this population in community settings. Further research is needed to inform the development of pain assessment tools specifically for use by paramedics in these settings.

Keywords:

pain assessment, dementia, paramedic students, residential aged care

Corresponding author: Peter V Lucas, p.v.lucas@utas.edu.au 


\section{Introduction}

Assessing pain in people with advanced dementia who have limited ability to communicate presents unique challenges to health care workers (1-3). This is particularly relevant for paramedics who are often the first point-of-contact for people experiencing pain in out-of-hospital settings $(4,5)$. Paramedics tend to be unfamiliar with the person they are assessing, usually lack a detailed medical history and, typically, have limited time to conduct a comprehensive pain assessment $(4,5)$.

Timely and thorough pain assessment is an important step in facilitating appropriate pain relief for people with dementia $(4,5)$. It has been argued that pain relief is one of the most important tasks paramedics perform for their patients $(6,7)$ and the provision of appropriate pain relief by paramedics is considered an indicator of quality care $(6,8)$.

The nature of paramedic practice is changing in many developed countries in response to demographic and epidemiologic transitions $(9,10)$. New positions such as extended care paramedics are being introduced to help reduce rates of unnecessary hospitalisation as part of an evolving concept of 'taking care to the patient, rather than taking the patient to care' (11). Extended care paramedics have been shown to provide positive outcomes for people in residential aged care facilities (RACFs), also known as 'nursing homes', by reducing disruption to patients and carers resulting from emergency department transfers (11). Paramedics' understanding of and responses to people with dementia experiencing pain may be an important component of such practice.

Estimates of the prevalence of pain among people living in RACFs vary markedly, as do estimates of the prevalence of pain in people with dementia. The Australian Pain Society estimates that as many as $40 \%$ of people living in RACFs experience difficulty in reporting pain due to cognitive and communicative impairments $(12,13)$. There is general consensus in the literature that pain is both under-diagnosed and under-treated in these populations (3). Moreover, there is evidence that pain in people with dementia is more likely to be undiagnosed and inadequately treated than in cognitively intact older people within RACFs $(14,15)$.

The complex and subjective nature of pain means that accurate assessment can be a barrier to effective management, particularly among those with cognitive impairments such as dementia $(2,4,5,16)$. Inadequately treated pain among this cohort is linked to both increased behavioural disturbances and reduced quality of life (2). Accurate pain assessment and treatment in community settings such as RACFs have been linked to reduced hospital admissions, which in turn is associated with increased morbidity and reduced life expectancy for people with dementia (17). As key mediators between community and hospital contexts, paramedics can potentially make a significant contribution to improving care for people with dementia (4), particularly those who are experiencing pain.

Systematic literature reviews of pain assessment tools for use with cognitively impaired adults failed to identify any instruments that have been clinically validated for use by paramedics $(4,5)$. Considering forecast increases in the prevalence of dementia (18), and the changing nature of paramedic practice discussed above, it is increasingly likely that paramedics will conduct pain assessments of people with dementia in community settings. Any pain assessment tool proposed for use by paramedics with a population of cognitively impaired older people must be practical and fit the operational requirements of paramedics who have limited time to spend with patients (5).

The Australian Pain Society evaluated several clinically validated and reliable pain scales designed for use with non-communicative adults, such as people with advanced dementia $(12,13)$. Two validated scales were recommended for use by health professionals in this population (12): the Abbey Pain Scale (19) and the Pain Assessment in Advanced Dementia Scale (PAINAD) (20). Both tools were deemed to have acceptable utility, validity and reliability, with the Abbey Pain Scale more widely accepted by RACF staff in Australia in published research $(12,13)$. Importantly for paramedic practice, both tools can be administered in a short period of time (13), hence they are potentially suited to the timeconstrained environment in which paramedics operate. This paper reports on an exploratory pilot study in which paramedic students on a 5-day clinical placement in RACFs administered the Abbey Pain Scale and PAINAD tools when assessing pain in residents with dementia. The primary aim of the research was to assess undergraduate student perceptions of the potential utility of two validated pain assessment tools for use by paramedics in community settings, including RACFs.

\section{Research methods}

\section{Research participants}

A cohort of final year undergraduate paramedic students $(n=31)$ participated in a 5 day aged care placement across four separate facilities as part of a supervised clinical RACF placement in Tasmania. Students who had used both assessment tools on their allocated resident during that time completed a survey exploring their views about various aspects of the tools.

\section{Research setting}

The research was conducted across four RACFs, ranging in size from 70 to 150 beds, in the Australian state of Tasmania during September and October 2013. Each facility offered high and low-care places for residents, and two of the facilities had secure units for people with moderate to severe behavioural and psychological symptoms of dementia. 


\section{Data collection and analysis}

While on placement, students used both the Abbey Pain

Scale and PAINAD tools to assess pain in residents living with dementia under the supervision of paramedic tutors and facility nursing staff who were familiar with the residents and their medical history. Students conducted pain assessments in addition to broader bio-psychosocial evaluations, with residents allocated by the facility as part of a structured interprofessional learning activity in collaboration with nursing and medical students. Previous findings of paramedic engagement with interprofessional learning in RACF settings have been reported elsewhere (21-23).

The number of assessments undertaken by paramedic students who completed the survey at the end of their clinical placement ranged from 2 to 6 . That is, students who completed surveys had used each tool at least once for assessing pain on their allocated resident. Some students reported having done assessments using both tools on up to three occasions during their placement. As the instructions accompanying the tools required residents to be observed while being moved, showered or during pressure area care, the number of assessments was dependent on both the availability of suitable residents for assessment and the workload of facility staff who could assist with moving the resident during observation.

Prior to using the tools in the clinical setting, students participated in a 2-hour education session with a paramedic tutor during which they learnt how to administer the tools. Additionally, students were provided with written instructions on the correct administration of the tools (see Appendix). A Likert-type scale employing scores from 0 to 10 was used to gauge the extent to which students perceived the tools were:

(a) simple to use; (b) accurate in assessing pain in people with dementia; (c) difficult to learn to use; (d) relevant to future role as paramedics; and (e) useful for assessing pain in cognitively impaired adults. The wording of these questions is listed in Table 1. There were also seven open-ended questions for which students could provide additional information in order to garner qualitative insights into the potential utility of the tools for paramedic practice in community settings.

Quantitative data from the student surveys were analysed using SPSS Version 20 (24). A descriptive analysis of the data was undertaken with comparisons being made between each of the pain tools used in relation to each of the criteria described above. Because the data was not normally distributed, non-parametric testing in the form of a Wilcoxon signed rank test for paired samples was used to identify any differences between student perceptions of the tools in relation to individual survey items, as well as the self-reported time taken to complete the assessment.

Qualitative data from the open-ended questions were analysed using a thematic methodology that employed an open coding process to identify latent themes within the data. These themes were then subjected to a more focused coding process that facilitated the development of inductive codes from which key analytic categories were developed (25).

\section{Ethics approval}

Ethics approval for the research was obtained from the Tasmanian Health and Medical Human Research Ethics Committee (No. H13449).

\section{Results}

\section{Quantitative data}

A total of 22 students completed a questionnaire about the Abbey Pain Scale and PAINAD tools at the end of their 5-day clinical placement in four RACFs during September to October 2013. Students who completed surveys were aged between 18 and 33 years, with a mean age of $21.95(S D=4.12)$; the majority were female $(62 \%)$. The remaining nine students in the cohort did not complete the questionnaire because they did not have the opportunity to assess residents with dementia who were experiencing pain.

The Abbey Pain Scale was rated slightly higher by students in terms of overall utility for assessing pain in cognitively impaired adults. However, on each of the individual characteristics, including simplicity of use, perceived accuracy, ease of learning and relevance to their future role as paramedics, the students rated PAINAD more highly than the Abbey Pain Scale. Mean scores of individual questions for both tools, which had a possible maximum score of 10 , are outlined in Table 1. As the question relating to how difficult it was to learn how to use the tools was an inverse measure, the scores for that item were reversed to attain consistency with the other scores.

Table 1. Mean scores of individual survey items

\begin{tabular}{|l|c|c|}
\hline & Abbey & PAINAD \\
\hline $\begin{array}{l}\text { To what extent was the scale a } \\
\text { simple pain assessment tool to } \\
\text { use? }\end{array}$ & $\begin{array}{c}7.29 \\
(\mathrm{SD}=1.82)\end{array}$ & $\begin{array}{c}8.05^{*} \\
(\mathrm{SD}=1.81)\end{array}$ \\
\hline $\begin{array}{l}\text { How accurate was the scale in } \\
\text { providing an assessment of the } \\
\text { resident's pain? }\end{array}$ & $\begin{array}{c}6.33 \\
(\mathrm{SD}=2.08)\end{array}$ & $\begin{array}{c}6.45 \\
(\mathrm{SD}=2.26)\end{array}$ \\
\hline $\begin{array}{l}\text { How difficult was it to learn to } \\
\text { use the scale to assess pain in } \\
\text { cognitively impaired residents? }\end{array}$ & 5.90 & $\begin{array}{c}7.32 \\
(\mathrm{SD}=2.59)\end{array}$ \\
\hline $\begin{array}{l}\text { To what extent will the scale be } \\
\text { relevant in your future role as a } \\
\text { paramedic? }\end{array}$ & 4.90 & $(\mathrm{SD}=2.77)$ \\
\hline $\begin{array}{l}\text { Overall, how useful is the scale } \\
\text { for assessing pain in cognitively } \\
\text { impaired adults? }\end{array}$ & $\begin{array}{c}6.33 \\
(\mathrm{SD}=1.98)\end{array}$ & $\begin{array}{c}6.274) \\
(\mathrm{SD}=1.78)\end{array}$ \\
\hline
\end{tabular}

* Higher scores indicate more positive sentiment towards a tool in relation to each statement 
Lucas: Pain assessment in older adults with dementia

Australasian Journal of Paramedicine: 2016;13(3)

Table 2. Time taken to administer pain scales

\begin{tabular}{|l|c|c|}
\hline & Abbey & PAINAD \\
\hline $\begin{array}{l}\text { Approximately how long did it } \\
\text { take to administer the scale? } \\
\text { (minutes) }\end{array}$ & 5.89 & 3.44 \\
$(\mathrm{SD}=4.75)$ & $(\mathrm{SD}=3.03)$ \\
\hline
\end{tabular}

Students reported that PAINAD took less time to administer than the Abbey Pain Scale. The mean estimated times for administering each of the tools are listed in Table 2. Subsequent statistical testing revealed that the reported time taken to complete each pain tool was significantly different $(Z=2.263 ; p=0.016)$.

\section{Qualitative data}

In addition to the rating scales in the questionnaires, students were asked to provide feedback on their perceptions and experiences of administering the two tools in several openended questions, with a view to discerning the potential utility of the tools for paramedic practice. Students were asked: a) what they liked most about each tool; b) what they disliked about each tool; c) how each of the tools could be improved for use in paramedic practice; and d) which tool they considered the most suitable for use by paramedics. These findings are reported in the following section.

\section{What paramedic students liked about the tools}

Several students reported they liked PAINAD because it was quick and simple to use. One commented, '[It's] quick, easy to use, practically what we do anyway, but subconsciously' (BPM29). Another made a similar comment, '[l's's] simple, quick, easy to remember and use. Most paramedics would observe these changes anyway as part of our physiological assessments and on transport to hospital' (BPM28). Others reported that they liked the descriptions that came with the tool (see Appendix) and felt this made it much easier to score than the Abbey Pain Scale. One student thought the consolability item was a good feature as 'it identifies the importance of reassurance and distraction for patients with pain' (BPM36). Others considered the fact that PAINAD rates pain on a scale of $0-10$ was another positive feature, as they felt this facilitated easier calculation of an overall score.

The most commonly reported positive feature of the Abbey Pain Scale that students liked was its capacity to assess physiological changes in residents with dementia: 'It assessed physiological change, not just subjective patient presentation' (BPM24). Some students considered the Abbey Pain Scale to have a broader range of categories to assess pain than the PAINAD scale while one reported they liked the tool because it, 'considers when pain relief was last given and that could affect overall score' (BPM21).

\section{What paramedic students disliked about the tools}

The most commonly reported feature of the PAINAD tool that students disliked was that it did not consider physiological changes. A student noted, 'It doesn't include physiological changes, but we would be reassessing vital signs/ physiological status anyway' (BPM28). Others remarked that the facial expression section could be misinterpreted with one student commenting that it was 'difficult to assess facial expression in stroke victims or depressed people [and] difficult to differentiate between inexpressive and sad' (BPM30). Another student reported they found the category 'breathing independent of vocalisation' 'a bit confusing' (BPM26).

For the Abbey Pain Scale the most commonly reported feature the students disliked was the requirement to wait for an hour before re-assessment of pain, as this was considered impractical for paramedics. Others considered the pain assessment categories 'mild', 'moderate' and 'severe' to be too subjective and this could potentially result in different interpretations of these measures, 'especially if it's the first encounter with a patient' (BPM25). Several students remarked the length of time the Abbey Pain Scale took to administer was a feature they disliked: 'the time taken to complete and the number of degrees of severity' (BPM29) was what one student reported as problematic for them.

\section{Potential adaptations to the tools for paramedic practice}

The most commonly suggested adaptation for the Abbey Pain Scale was for a shorter timeframe between re-assessment. In the context of paramedic assessment a student stated, 'Having to wait an hour is not practical for reassessment. Reassessment after a few minutes or after interventions would be better' (BPM28). Some students felt that the section on behavioural changes could be removed, as this requires prior knowledge of the patient that would often not be available to paramedics, and this would speed up the assessment process.

For PAINAD, one recurring theme from students was that it should include a section that took into consideration physiological changes. One student suggested 'adding more examples to the categories, such as facial expression, [as it's] difficult to determine whether the facial expression provides an indication as to the severity very well' (BPM36).

\section{Which tool is most suitable for use by paramedics?}

Two-thirds of the students surveyed in this exploratory study $(n=14)$ who identified a preferred tool, and provided reasons for this, considered PAINAD to be more suitable for use by paramedics. The reasons given for this preference included that it was, 'easier to rate responses' (BPM26) and 'does not require us to assess behavioural changes, it just assesses body language [and is] easier to apply pre-hospital as we only see the patient for a short time' (BPM36). A typical reason for rating PAINAD more highly was 'because it's a quick and effective assessment of pain' (BPM42). Those who preferred the Abbey Pain Scale indicated they felt it was 'a more comprehensive tool' (BPM38) with 'more variables' (BPM24) and 'provides greater detail under each behavioural category [and] includes physiological changes such as blood pressure and skin as indicators of pain' (BPM43). On the other hand one student responded 'none of them [are suitable], as they are too subjective' (BPM40). 


\section{Discussion}

The objective of this exploratory pilot study was to ascertain undergraduate paramedic students' views of two clinically validated pain assessment scales recommended by the Australian Pain Society for use with non-communicative adults. The research did not seek to evaluate the psychometric properties of the tools as both have already been validated for the purpose of assessing pain in noncommunicative older people. The results from this study suggest the two scales hold promise for assisting in pain assessment of cognitively impaired older patients when paramedics are called out to such individuals in community settings.

The need for such a tool in paramedic practice has previously been identified in the literature, however, further research into potential tools was called for $(4,5)$. The findings from this exploratory study support the need for such a tool while identifying strengths and weaknesses with two existing tools approved for use by the Australian Pain Society. One issue of concern in the results from the quantitative component of the research was that students did not rate either tool highly in terms of relevance to their future role as paramedics. This has significant implications for paramedic education and suggests that students may not fully appreciate the changing health care landscape in which they will be working (26).

If these tools were to be validated for use by paramedics through appropriate clinical trials they could potentially reduce unnecessary suffering and improve quality of life for people with dementia and other forms of cognitive impairment. As discussed above, undiagnosed and untreated pain has been recognised as a problem in this population (3). Where pain assessment has been routinely undertaken, followed by analgesia where appropriate, a corresponding decrease in pain related behaviours has been observed (27). With the changing nature of paramedic practice (9), where more extended care paramedics will be providing treatment and care to older people in community settings (11) the addition of tools that would enable timely and accurate assessment of pain could, potentially, reduce unnecessary transport to emergency departments and the multiple issues associated with this.

\section{Limitations of this study and suggested future research}

This paper reports on an exploratory pilot study that is the first of its type involving paramedic students using validated tools to assess pain in non-communicative adults. The research did not seek to determine inter-rater reliability between students, or to compare students' scores with those assessed by experienced aged care staff. Both tools have already been validated for the purpose of assessing pain in non-communicative older persons, and the primary aim of this exploratory study was to get the perspective of a cohort of students on clinical placement in RACFs about the tools' potential for use by paramedics.

As with any study this research has limitations, the small sample size being foremost among them. Another limitation was that not all students who completed the survey had the opportunity to use both tools the same number of times. The number of assessments could not be strictly controlled due to the structure of the clinical placement, with students' allocated specific residents before the placement began and not all residents allocated to students as part of the interprofessional learning component of the placement had dementia or poorly managed pain. As has been identified elsewhere, RACFs can be challenging teaching and learning environments due to staffing and workload issues (28) as well as RACF staff having a limited understanding of paramedics scope of practice (22). Ensuring all students conducted an equal number of assessments would have made a project of this nature untenable by further increasing staff burden, however, such limitations should not detract from the identification of two potentially important clinical tools that could augment the role of paramedics in the field.

We recommend an expanded comparative analysis of these tools across multiple RACFs and other community locations with representative samples of paramedic students, RACF staff, paramedic tutors and older non-communicative adults with known pain issues. Alternatively, development of a tool specifically for use by paramedics through appropriately designed clinical trials would serve to augment paramedics' changing role and extended scope of practice within contemporary health care systems. Future studies could also consider investigating other important pain management topics such as students 'intention to treat' based on any pain assessments they conduct.

\section{Conclusion}

Appropriate assessment and management of pain in people with dementia is an important indicator of quality care, as well as a fundamental human right (6). With increasing rates of dementia in an ageing population and growing emphasis on ageing in place (29), the ability of paramedics to accurately assess and treat pain in this population is critical. Indeed, training of all health care professionals working with older people with cognitive impairments such as dementia should include instructions on the use of appropriate pain assessment tools (30). Further trials of the two pain assessment tools used in this exploratory pilot project are crucial for ensuring paramedics are adequately equipped to meet the challenges posed by these demographic and epidemiologic transitions. 


\section{Acknowledgement}

The authors wish to acknowledge the support provided by staff at the residential aged care facilities in which the clinical placements were conducted for making this research project possible.

\section{Conflict of interest}

The authors declare they have no competing interests. Each author of this paper has completed the ICMJE conflict of interest statement.

\section{References}

1. Cadogan MP. Assessing pain in cognitively impaired nursing home residents: The state of the science and the state we're in. J Am Med Dir Assoc 2003:4:50-1.

2. Corbett $A$, Husebo B, Malcangio $M$, et al. Assessment and treatment of pain in people with dementia. Nat Rev Neurol 2012;8:264-74.

3. Lichtner V, Dowding D, Esterhuizen P, et al. Pain assessment for people with dementia: a systematic review of systematic reviews of pain assessment tools. BMC Geriatr 2014;14:138.

4. Harvey $\mathrm{C}$. Is there scope for an observational pain scoring tool in paramedic practice? Journal of Paramedic Practice 2014;6:84-8.

5. Lord B. Paramedic assessment of pain in the cognitively impaired adult patient. BMC Emerg Med 2009;9:20.

6. Lord B, Nicholls T. A brief history of analgesia in paramedic practice. Journal of Paramedic Practice 2014;6:400-6.

7. McLean SA, Maio RF, Domeier RM. The epidemiology of pain in the prehospital setting. Prehosp Emerg Care 2002;6:402-5.

8. Lord B, Deveson M. Assessment and management of chronic pain in adults: implications for paramedics. Journal of Paramedic Practice 2011;3:166-72.

9. Arendts G, Lowthian J. Demography is destiny: An agenda for geriatric emergency medicine in Australasia. Emerg Med Australas 2013;25:271-8.

10. Hagihara A, Hasegawa M, Hinohara Y, Abe T, Motoi M. The aging population and future demand for emergency ambulances in Japan. Intern Emerg Med 2013;8:431-7.

11. Health Workforce Australia. Extended Care Paramedics 2014. Available at: www.hwa.gov.au/our-work/expandedscopes-practice-program/extended-care-paramedics [Accessed 16 June 2014].

12. Abbey J. Putting pain scales to the test. Aust Nurs J 2007;14:43.

13. Goucke R, Scherer S, Katz B, Gibson S, Farrell M, Bradbeer M. Pain in residential aged care facilities: management strategies. Sydney, NSW, Australian Pain Society. 2005 s: Management strategies. Sydney, NSW,
Australian Pain Society. 2005.

14. Husebo BS, Strand LI, Moe-Nilssen R, BorgeHusebo S, Aarsland D, Ljunggren AE. Who suffers most? Dementia and pain in nursing home patients: a cross-sectional study. J Am Med Dir Assoc 2008:9:427-33.

15. Reynolds KS, Hanson LC, DeVellis RF, Henderson M, Steinhauser KE. Disparities in pain management between cognitively intact and cognitively impaired nursing home residents. J Pain Symptom Manage 2008;35:388-96.

16. McAuliffe L, Nay R, O'Donnell M, Fetherstonhaugh D. Pain assessment in older people with dementia: literature review. J Adv Nurs 2009;65:2-10.

17. Sampson EL, Blanchard MR, Jones L, Tookman A, King M. Dementia in the acute hospital: prospective cohort study of prevalence and mortality. Br J Psychiatry 2009;195:61-6.

18. World Health Organization, Alzheimer's Disease International. Dementia: A Public Health Priority. Geneva: World Health Organization, 2012.

19. Abbey J, Piller N, De Bellis A, et al. The Abbey pain scale: a 1-minute numerical indicator for people with end-stage dementia. Int J Palliat Nurs 2004;10:6-13.

20. Warden V, Hurley AC, Volicer L. Development and psychometric evaluation of the Pain Assessment in Advanced Dementia (PAINAD) scale. J Am Med Dir Assoc 2003:4:9-15.

21. Lucas P, McCall M, Lea E, et al. Clinical placements in residential care facilities part 1: positive experiences. Journal of Paramedic Practice 2013;5:400-6.

22. Lucas $P, M c C a l l ~ M$, Lea $E$, et al. Clinical placements in residential care facilities part 2: negative experiences. ibid. 2013;5:462-8.

23. Lucas PV, McCall MJ, Ecclestone C, et al. Prioritising the development of paramedic students' interpersonal skills. ibid. 2015;7:245.

24. IBM Corp. IBM SPSS Statistics for Windows, Version 20.0. Armonk, New York: IBM Corp; 2011.

25. Ezzy D. Qualitative Analysis: Practice and innovation. Crows Nest: Allen \& Unwin; 2002.

26. Williams B, Onsman A, Brown T. The changing Australian healthcare landscape: implications for paramedics. Journal of Paramedic Practice 2010;2:580-4.

27. Cohen-Mansfield J, Lipson S. The utility of pain assessment for analgesic use in persons with dementia. Pain 2008;134:16-23.

28. Robinson AL, Andrews-Hall S, Fassett M. Living on the edge: issues that undermine the capacity of residential aged care providers to support student nurses on clinical placement. Aust Health Rev 2007;31:368-78.

29. Marek KD, Popejoy L, Petroski G, Mehr D, Rantz M, Lin W-C. Clinical outcomes of aging in place. Nurs Res 2005;54:202-11.

30. Royal College of Physicians, British Geriatrics Society, and British Pain Society. The assessment of pain in older people: national guidelines. Concise guidance to good practice series, No 8. London: RCP; 2007. 